\title{
Acute neurological care in north-east Germany with telemedicine support (ANNOTeM): protocol of a multi-center, controlled, open-label, two-arm intervention study
}

J. E. Weber ${ }^{1,2,3^{*}}$ (D) A. Angermaier ${ }^{4}$, K. Bollweg ${ }^{1}$, H. Erdur ${ }^{1,2}$, S. Ernst ${ }^{3,6}$, A. Flöel ${ }^{4,5}$, C. Gorski ${ }^{6}$, F. I. Kandil ${ }^{6,7}$, S. Kinze ${ }^{8}$, K. Kleinsteuber ${ }^{6}$, T. Kurth $^{6}$, I. Schmehl ${ }^{8}$, S. Theen ${ }^{6}$, M. Endres ${ }^{1,2,9,10,11}$, H. J. Audebert ${ }^{1,2}$ and for the

ANNOTeM-consortium

\begin{abstract}
Background: Both diagnosis and treatment of neurological emergencies require neurological expertise and are time-sensitive. The lack of fast neurological expertise in regions with underserved infrastructure poses a major barrier for state-of-the-art care of patients with acute neurological diseases and leads to disparity in provision of health care. The main purpose of ANNOTeM (acute neurological care in North East Germany with telemedicine support) is to establish effective and sustainable support structures for evidence based treatments for stroke and other neurological emergencies and to improve outcome for acute neurological diseases in these rural regions.
\end{abstract}

Methods: A "hub-and-spoke" network structure was implemented connecting three academic neurological centres ("hubs") and rural hospitals ("spokes") caring for neurological emergencies. The network structure includes (1) the establishment of a 24/7 telemedicine consultation service, (2) the implementation of standardized operating procedures (SOPs) in the network hospitals, (3) a multiprofessional training scheme, and (4) a quality management program. Data from three major health insurance companies as well as data from the quality management program are being collected and evaluated. Primary outcome is the composite of first time of receiving paid outpatient nursing care, first time of receiving care in a nursing home, or death within 90 days after hospital admission.

Discussion: Beyond stroke only few studies have assessed the effects of telemedically supported networks on diagnosis and outcome of neurological emergencies. ANNOTeM will provide information whether this approach leads to improved outcome. In addition, a health economic analysis will be performed.

(Continued on next page)

\footnotetext{
* Correspondence: joachim.weber@charite.de

${ }^{1}$ Klinik und Hochschulambulanz für Neurologie, Charité - Universitätsmedizin Berlin, Hindenburgdamm 30, D-12203 Berlin, Germany

${ }^{2}$ Center for Stroke Research Berlin (CSB), Charité - Universitätsmedizin Berlin, Berlin, Germany

Full list of author information is available at the end of the article
}

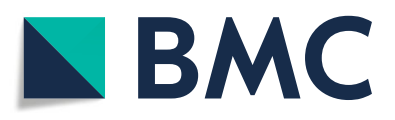

(- The Author(s). 2020 Open Access This article is licensed under a Creative Commons Attribution 4.0 International License, which permits use, sharing, adaptation, distribution and reproduction in any medium or format, as long as you give appropriate credit to the original author(s) and the source, provide a link to the Creative Commons licence, and indicate if changes were made. The images or other third party material in this article are included in the article's Creative Commons licence, unless indicated otherwise in a credit line to the material. If material is not included in the article's Creative Commons licence and your intended use is not permitted by statutory regulation or exceeds the permitted use, you will need to obtain permission directly from the copyright holder. To view a copy of this licence, visit http://creativecommons.org/licenses/by/4.0/. The Creative Commons Public Domain Dedication waiver (http://creativecommons.org/publicdomain/zero/1.0/) applies to the data made available in this article, unless otherwise stated in a credit line to the data. 
(Continued from previous page)

Study registration: German Clinical Trials Register DRKS00013067, date of registration: November 16 th, 2017, URL: http://www.drks.de/DRKS00013068

Keywords: ANNOTeM, Emergency medicine, Health care research, Neuro acute units, Quality management, Stroke / Neurological disease, Telemedicine,

\section{Background}

The implementation of stroke units [1] along with the advent of intravenous thrombolysis [1] as well as thrombectomy of large vessel occlusion [2] has led to a huge leap in evidence-based acute stroke therapy. However, treatment effects are time-sensitive and depend on swift application of specific therapies. This is not only true for stroke but also for other neurological emergencies like seizures, meningoencephalitis, and spinal cord injury, where fast and effective diagnostication and treatment have been shown to improve outcome [3-5]. Neurological emergencies are a major cause of functional dependence [6]. Hospitals with emergency departments but without a neurology sercive may struggle to provide up-to-date care for patients with neurological emergencies. This applies particularly to rural and other regions with underserved infrastructure. Unfortunately, most neurological diseases peak at higher age, which means that rural areas with typically elderly populations face a particular challenge in providing appropriate care.

Telemedicine networks offer the potential for providing neurological expertise for emergency departments without expert neurologists [7]. In fact, neurological diseases can be adequately assessed via audiovisual examination because typical neurological deficits like palsies, disturbances of speech or consciousness can be reliably captured via video stream $[8,9]$ and brain imaging is easily transferable digitally.

Telestroke networks have been developed as a blueprint for teleneurological treatment during recent years. Treatment of acute stroke using telemedicine resulted in more frequent and appropriate application of intravenous thrombolysis $[10,11]$ and was associated with improved outcome [12]. What is more, "telestroke" proved to be cost-effective [13] making stroke one of the most frequent indications for telemedicine in several countries (Refs als from US, Germany etc).

\section{Aims}

ANNOTeM (acute neurological care in North-East Germany with telemedicine support) aims to improve health care by use of telemedicine for acute timedependent neurological diseases beyond stroke, in particular coma/status epilepticus, meningoencephalitis, and spinal cord injury, and therefore establish sustainable structures for implementation of evidence based treatments in underserved areas in North-East Germany.

\section{Methods/design Hypothesis}

A multi-component intervention with telemedicine support for neurological emergency care extends time to death or first time of receiving paid outpatient nursing care or first time of receiving care in a nursing home within 90 days after hospital admission.

\section{Study design and setting}

ANNOTeM is a multi-center, controlled, open-label, twoarm intervention study. Primary and secondary outcomes will be evaluated for pre- and post-implemention of the network. The ANNOTEM intervention started in November 2017 and is expected to run until the end of July 2020.

\section{Conventional care}

Community hospitals, except one without a neurology department, were included in the teleneurological network if they had treated a significant number of at least 50 patients per year with main discharge diagnosis of an acute neurological disease. During the control period, patients had usually been admitted to the internal medicine department and had been seen by a neurologist the following day at the earliest. Typically, management of acute neurological diseases had not been based on standardized operating procedures (SOP) in these hospitals prior to the project.

\section{Intervention}

\section{Structure of the network}

The teleneurological network ANNOTeM consists of a consortium of four neurocenters (Charité -Universitätsmedizin Berlin, Universitätsmedizin Greifswald, Unfallkrankenhaus Berlin, Epilepsieklinik Tabor) with neurological and epileptological expertise, three health insurance companies (Allgemeine Ortskrankenkasse Nordost, Potsdam (AOK Nordost), Barmer Ersatzkasse, Wuppertal (BARMER), Techniker Krankenkasse, Hamburg (TK)), one technology partner specialized on telemedicine communication products (MEYTEC, Werneuchen, Germany) and the Institute of Public 
Health, Charité -Universitätsmedizin Berlin,as evaluating institute. The consortium is led by the Charité - Universitätsmedizin Berlin. The clinical centers provide teleneurological care to 11 regional hospitals in the northeastern federal states of Germany $(2$ in Mecklenburg-Western Pomerania, 8 in Brandenburg and one in Saxony-Anhalt). One regional hospital runs its own neurological department with a certified regional Stroke Unit. In the ten hospitals, the "Neuro Acute Units" were set up as part of the intervention and managed by the respective departments of internal medicine in nine hospitals, whereas in the remaining hospital the "Neuro Acute Unit" is managed as part of an interdisciplinary intensive care unit. In addition, the network cooperates with other neurological, neuroradiological and neurosurgical facilities in the region.

\section{Neuro-acute-units}

The structure of the "Neuro-Acute-Units" is based on the concept of the German Tele-Stroke-Units [14] and aims at specialized treatment for a broader spectrum of acute neurological diseases. In addition to the standards already defined for Tele-Stroke Units, quality management, training, courses, and bed-side visits will be extended to the management other acute neurological diseases considered as part of the intervention for physicians, nurses and therapists.

\section{Implementation of standardized operating procedures}

Standardized operating procedures (SOP) for management of acute stroke, epileptic seizures/epilepsy, meningitis/encephalitis, and acute spinal cord injury were developed by consensus of the four neurocenters [15, 16]. These SOPs are generally based on the guidelines of the German Neurological Society (Deutsche Gesellschaft für Neurologie, DGN) und Joint Workgroup of German Medical Societies (Arbeitsgemeinschaft der Wissenschaftlichen Medizinischen Fachgesellschaften, AWMF), but had to be adapted in some aspects with regard to the telemedicine setting. Furthermore, they undergo regular updates according to the evolving scientific evidence. Before initiating teleconsultations, each SOP was presented to each team of the network hospitals followed by a discussion of their implementation in clinical routine.

\section{Quality management}

With start of the network, each cooperating community hospital started documentation of quality indicators within the quality assessment for stroke in Northwest Germany (Qualitätssicherung Schlaganfall Nordwestdeutschland, based on the Arbeitsgemeinschaft Deutschsprachiger Schlaganfall Register (ADSR)). The ANNOTeM network developed additional quality assessment instruments for other acute neurological diseases (epilepsy, meningitis/ encephalitis, and spinal cord injury) for their use in the regional hospitals.

In addition to quality assessment, regular meetings on treatment quality are held in the cooperating regional hospitals with mutual discussion of the proportions of patients presented via telemedicine, documentation rates as well as the SOP-adherence and interhospital transfers of patients.

\section{Teleneurological consultations}

Teleneurological consultations are provided by physicians who are experienced in stroke treatment and meet the standard of board certified neurologists for diagnosis and treatment of neurological emergencies. The consultation service is staffed $24 / 7$ by a full-time physician. All physicians working in the telemedicine service receive a practice training for the technical system as well as for the procedures of teleneurological examination and treatment of patients. The cooperating regional hospitals can present all patients with acute neurological symptoms in teleconsultation. For this purpose, a hotline is used to contact the telemedicine centers. The patient is examined via video conference with remote-controlled camera together with the onsite physician. In addition, there is a connection to the imaging data systems of the cooperating regional hospitals. This can be used to forward any computer tomography (CT) or magnetic resonance imaging (MRI) images to a central Picture Archiving and Communication System (PACS), accessible from the teleneurology hubs. The data transfer in ANNOTeM is realized as a point-to-point connection between the respective active neurocentres and the regional cooperation hospitals (see Fig. 1). All connections are encrypted according to state-of-the-art data protection requirements (GDPR, General Data Protection Rules). Data are stored on secured servers with access restricten to staff of the medical project management. Video streams are not stored. After each consultation, a written statement with recommendations for acute treatment and further diagnostic procedures is sent electronically (PDF) to the regional hospital and printed automatically. During the monitoring on the neuroacute unit, a board certified neurologist sees the patient during ward rounds on a daily basis.

\section{Transfer management}

Patients who need specialized treatment beyond the capacities of the implemented "Neuro-Acute-Units" (in particular patients with need for neuroradiological or neurosurgical treatment, not being available in regional network hospitals) are transferred to the nearest suitable hospital. In order to avoid delays in the referral hospital, point-to-point connections have been established between the telemedicine centres and the various referral 


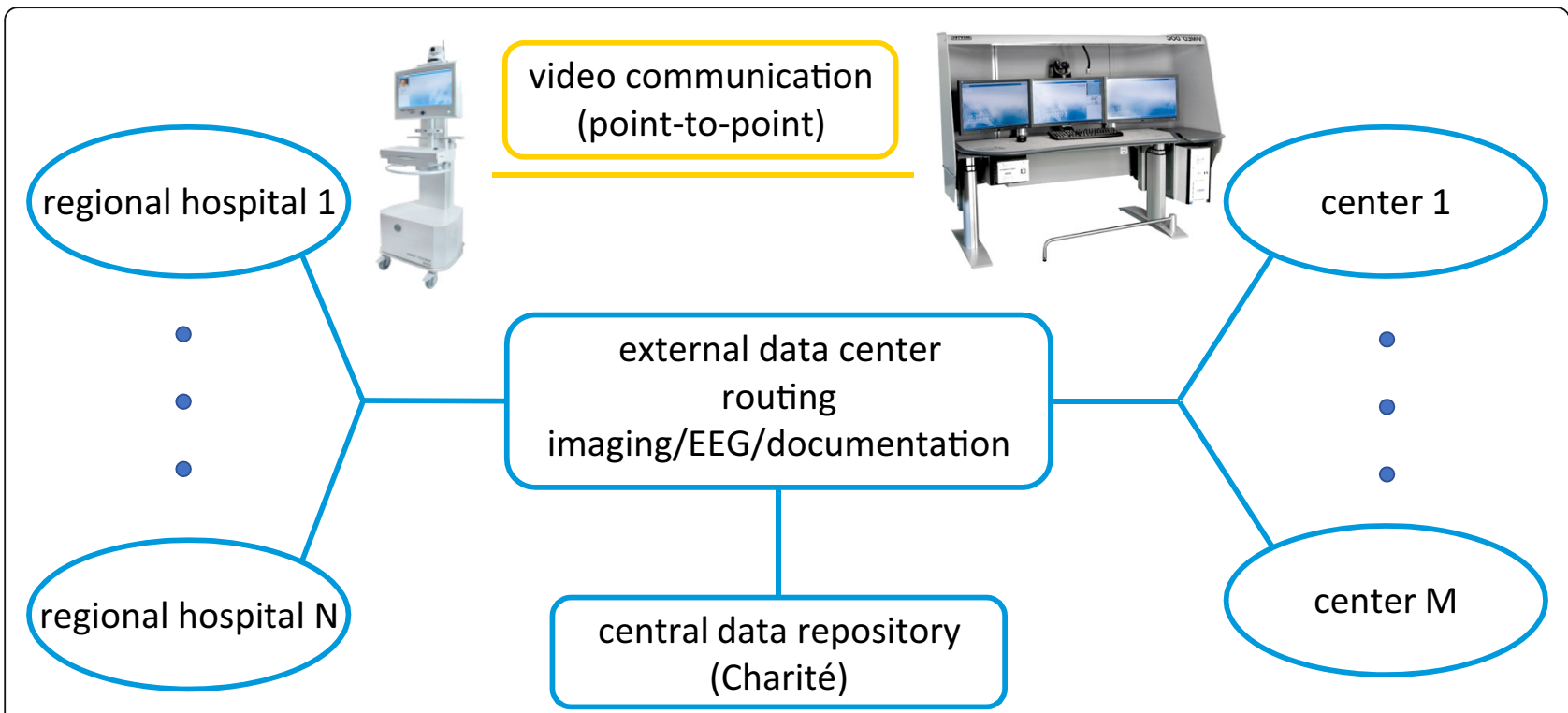

Fig. 1 Representation of data flows in the telemedical network ANNOTeM. The video data is transmitted as a stream using a point-to-point connection, while the documentation and other examination data are stored in a central repository. With permission of MEYTEC GmbH, the telemedical equipment used in ANNOTeM is shown (left: VIMED TELEDOC, right: VIMED DOC)

hospitals, allowing the transmission of imaging data collected in network hospitals. In addition, referral hospitals report data on processes and in-hospital outcomes for the respective patients to the coordinating center.

\section{Study population \\ Inclusion criteria}

To determine the composite primary outcome of time to death or first-time care at home or paid care support for the first time within 90 days after the index event, we will use routine data from three cooperating health insurance companies (AOK Nordost, BARMER, TK). For this purpose, eligible study participants must (1) have continuous health insurance with one of these health insurers during the evaluation period, (2) be admitted during the intervention or control period to one of the cooperating regional hospitals, (3) have a neurological diagnosis (International Classification of Diseases (ICD)10 main diagnosis) such as stroke (I60-64), status epilepticus or impaired level of consciousness (G41, R40), meningitis /encephalitis (A82.0-2, A85-87, G00-03, G06-09), acute spinal cord injury (S14, S24, S34, G82), (4) and be over 18 years of age of both sexes.

We had originally planned to include patients diagnosed with traumatic brain injury (S06-07) in an earliert version of the study protocoll. However, since patients with traumatic brain injury are only rarely managed by our project partners in the participating regional hospitals they are not included in the primary study population, but rather as a companion study population in a sensitivity analysis.

\section{Intervention group (post-implementation)}

The intervention group consists of patients treated in one of the eleven cooperating regional hospitals diagnosed with any of the acute neurological diseases (stroke, seizures/status epilepticus, meningoencephalitis, and spinal cord injury) defined in inclusion criteria between November 2017 and July 2020.

\section{Control group (pre-implementation)}

Patients included in the control group were admitted and treated with one of the above mentioned acute neurological diseases in the same cooperating regional hospitals between November 2014 and May 2017, i.e. before start of the ANNOTeM implementation.

\section{Comparisons \\ Comparison of control and intervention period in cooperating regional hospitals}

Patients with a diagnosis of a defined acute neurological disease and admitted to the cooperating regional hospitals during the intervention period from November 2017 to July 2020 are compared with patients of the same diagnosis group during the control period from November 2014 to May 2017 before the start of the intervention.

\section{Comparison with non-participating hospitals}

In order to investigate whether differences in outcome in the cooperating hospitals may be caused by temporal trends, the composite primary outcome will also be analysed for the same two periods in patients fulfilling the 
identical inclusion criteria but being treated in hospitals of the same region that do not participate in the network (non-network community hospitals). The non-network hospitals were matched according to geographical location, size and amount of acute neurological cases per year. Data for this comparison are also provided by the three insurance companies.

\section{Study objectives}

\section{Primary outcomes}

The composite primary composite outcome is time to 1) death, 2) first-time nursing home care or, 3) paid outpatient nursing care for the first time within 90 days after the index event, whichever occurs first.

\section{Secondary outcomes}

Secondary outcomes are: 1) Treatment proportions for evidence-based therapies such as Stroke Unit treatment, intravenous thrombolysis, endovascular thrombectomy, hemicraniectomy; 2) Hospital transfers; 3) Mortality (Cox regression); 4) Nursing home care; 5) Costs for acute inpatient treatment, hospital transfers, rehabilitation treatment and nursing care.

\section{Sample size calculation}

Sample size was calculated on the assumption that the implementation of the ANNOTeM concept in the cooperating regional hospitals reduces the rate of dependency from $39 \%$ in the control period to $32 \%$ in the intervention period, i.e. a relative reduction by one fifth. The assumptions are based on the results of the TEMPiS trial [13].

Patient recruitment started in November 2017 and will run to 31 July 2020. According to the annual case numbers, at least 1820 patients per group with defined inclusion criteria are expected in the eleven cooperating regional hospitals during the intervention period. This sample size ensures a power of $80 \%$ (corresponding to a beta of 0.20 ) and an alpha of $5 \%$ for a two-sided test. Case size calculation was performed in nQuery Advisor 7.0.

\section{Study time scale}

Eligible patients who were admitted to the cooperating hospitals from November 16th 2014 to May 31st 2017 will form the control group. After an implementation period of the ANNOTeM concept, patient recruitment was started in November 2017 in the first intervention hospitals and will continue until July 2020 for primary analysis with 90 days' follow-up (for details see Table 1 ). The same periods will be used for the evaluation of time trends in non-network hospitals.

An interim analysis without significance testing is planned after completion of $50 \%$ of patient follow-ups in order to inform decision makers about the progress of the scientific evaluation and options for transition into regular care; after the last follow-up is completed end of October 2020, the final analysis is expected in January 2021 (Fig. 2).

\section{Statistical analyses Primary analysis}

Because health insurance data will be provided anonymously, we cannot determine the effect of telemedicine consultation on an individual patient level. Rather, we will evaluate the effects of the ANNOTeM concept in clusters. The primary outcome will be analysed as the time from the index visit to the occurrence of the compound outcome as described above. Separate Cox models will be fitted for eligible patients insured at the cooperating health insurance companies, admitted and treated in both, the pre-implementation period and the intervention period. Cox models will comprise sex $(\mathrm{m} / \mathrm{f}$ only), age and co-morbidity $(\mathrm{Y} / \mathrm{N})$ as covariates. Hazard rate ratios will be used to compare time-to-compound event between the two periods. All tests will be

Table 1 Start of implementation and recruitment of patients as well as end of recruitment of the intervention hospitals in the study

\begin{tabular}{llll}
\hline Intervention hospitals & Start of implementation period & start of recruitment & end of recruitment \\
\hline BER & $10 / 04 / 2017$ & $01 / 02 / 2018$ & $07 / 31 / 2020$ \\
LUD & $07 / 02 / 2017$ & $11 / 16 / 2017$ & $07 / 31 / 2020$ \\
NAU & $07 / 08 / 2017$ & $11 / 16 / 2017$ & $07 / 31 / 2020$ \\
LUC & $08 / 20 / 2017$ & $11 / 18 / 2017$ & $07 / 31 / 2020$ \\
KYR & $12 / 11 / 2017$ & $11 / 16 / 2018$ & $07 / 31 / 2020$ \\
STR & $12 / 23 / 2017$ & $03 / 23 / 2018$ & $07 / 31 / 2020$ \\
TEM & $12 / 30 / 2017$ & $03 / 30 / 2018$ & $07 / 31 / 2020$ \\
RAT & $10 / 06 / 2018$ & $01 / 04 / 2019$ & $07 / 31 / 2020$ \\
UEC & $10 / 16 / 2018$ & $01 / 14 / 2019$ & $07 / 31 / 2020$ \\
GAR & $11 / 12 / 2018$ & $07 / 10 / 2019$ & $07 / 31 / 2020$ \\
PRE & $10 / 16 / 2018$ & $01 / 14 / 2019$ & $07 / 2020$ \\
\hline
\end{tabular}




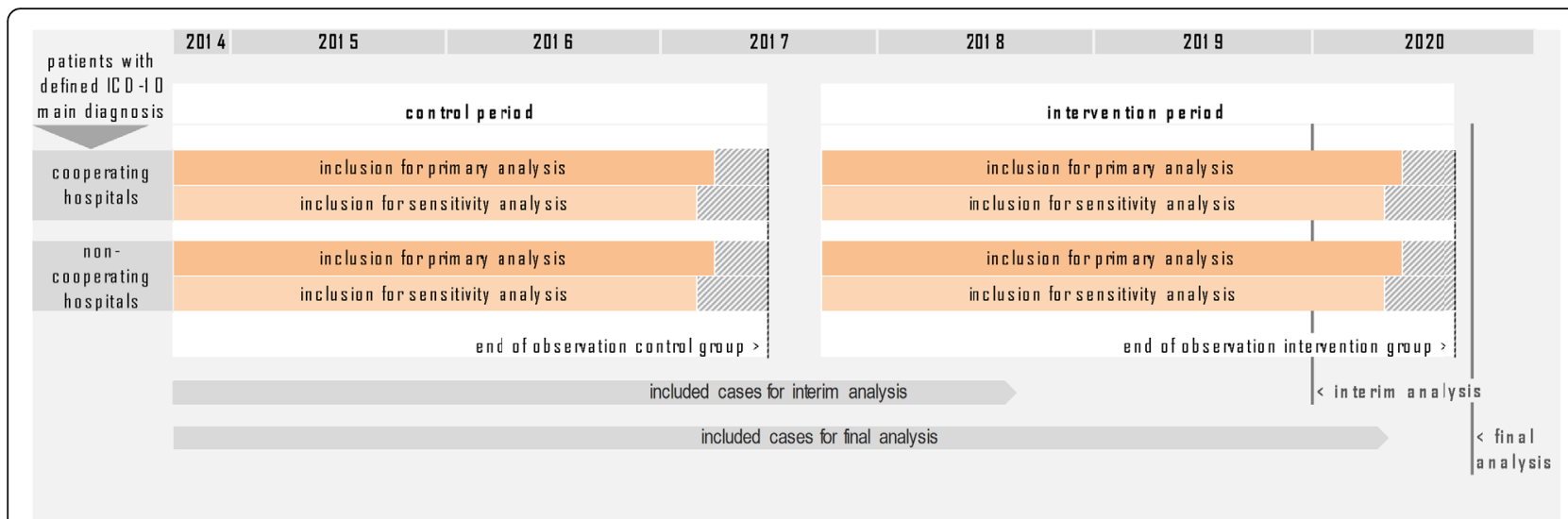

Fig. 2 Illustration of the study design of ANNOTeM over time (Study time scale). For the intervention group there is a comparison group both during the intervention period and during the historical reference period

performed at a significance level of alpha $=0.05$, adjusted for two-sided testing and the $\mathrm{k}=6$ tests of the primary and secondary analysis. All tests will be performed using the statistics package ' $R$ ' (version 3.6).

\section{Secondary analysis}

The secondary analysis compares the survival rates obtained in the primary for the network clinics with the group of non-cooperating hospitals using the log-rank-test for $2 \times$ 2 factorial designs (control vs intervention period and network vs non-network hospitals). A treatment effect will be revealed by a significant interaction in favour of the network clinics during the intervention period.

\section{Sensitivity analysis}

In a sensitivity analysis, we extend the follow-up of the composite primary outcome to 120 days after the index event. Secondly, we perform separate analyses for the outcomes death, first-time nursing home care and paid nursing care for the first time, which together make up the composite endpoint in the primary analysis. Thirdly, we perform a subgroup analysis of the composite primary outcome for following groups: patients with stroke vs other defined acute neurological diseases; patients with vs without nursing care before the index event; patients in cooperating hospitals with vs without neurological departments or telemedicine support before the start of the ANNOTeM intervention. Finally, we investigate patients in the cooperating hospitals with traumatic brain injuries who cannot be included in the main intervention group as a companion study population.

\section{Data collection, management, and analysis Data collection methods}

The health insurance data for determining the defined outcomes are provided by the cooperating statutory health insurance companies (AOK Nordost, BARMER,
TK) in accordance with the defined inclusion criteria. Within the framework of ANNOTeM, pseudonymised personal data of the health insurances will be transmitted in accordance with $\mathbb{\$ 7 5}$ Social Security Code V to a third trust party, which forwards the data in anonymised form to the evaluating institute. This forwarding has been approved by the supervisory authorities of the participating health insurance companies.

In addition to the account data for identifying the defined outcomes and costs, basic data on age and sex, insurance status and paid nursing care or nursing home care are provided at the beginning of observation.

\section{Data management}

The manual for data management was approved by TMF (Technologies, Methods and Infrastructure for Networked Medical Research) and the data protection officer of the Charité.

\section{Discussion}

Ageing populations along with structural deficits in underserved regions of North-East Germany [17] with an increasing shortage of medical specialists are major challenges in providing state-of-the-art treatment for acute neurological patients. At the same time, there is a need for comprehensive access to new, evidence-based therapies and standardized operating procedures for neurological emergencies in community hospitals, mostly without a neurology department. New initiatives to combine medical expertise with the use of modern infrastructural components have been established within the framework of telemedicine networks. So far, evidence for improving the quality of care is only available from acute telestroke projects. Hence, there is a need for large-scale studies that examine the benefits of such networks for outcomes and cost-effectiveness. ANNOTeM evaluates clinical outcomes for patients with acute 
neurological diseases including but not exclusively stroke treated with telemedicine support. ANNOTeM aims to establish and sustainably improve regional structures by continuous knowledge transfer to all professional groups involved in the care of acute neurological patients in the regional hospitals by continuous training and the introduction of standardized processes.

We acknowledge several limitations of the study: Changes in the care of patients with effects on the primary outcomes measured here may occur independently of study design through health policy measures and health care innovations. This can result in an overestimate of improved care for the intervention group compared to the pre-implementation control group. In order to control for this possible time trend, we have introduced a parallel control group of patients treated in non-network hospitals. A further limitation is that the definition of part of the composite outcome is based on routine data of the health insurances. It is possible that patients or their relatives do not apply for home care support provided by the health system even when they have a respective need of assistance. In addition, the decision for home care also depends, among other things, on the available social support at home or personal preferences. However, previous analyses have shown that the parameters of care support and home care correlate well with the degree of disability [18]. The approach taken in this project will be evaluated in the setting of the German health care system and results can therefore not be generalized.

On the other side, the study has several important strengths. First, the total number of patients treated in the hospitals with regard to the main diagnoses is recorded on the basis of health insurance data. Thus, the bias of patient selection by informed consent procedures often present in clinical studies is minimized. Second, study outcomes are assessed independently. Third, the combination of data collected within the telemedicine consultations along with quality management of the health insurance data provide the opportunity to evaluate the effects with a direct link to the health care context.

\section{Study status}

The intervention started in November 2017 and results are expected in January 2021.

\section{Abbreviations \\ AWMF: Joint Workgroup of German Medical Societies (Arbeitsgemeinschaft der Wissenschaftlichen Medizinischen Fachgesellschaften); CT: Computer tomography; DGN: German Neurological Society (Deutsche Gesellschaft für Neurologie); GDPR: General Data Protection Regulation; ICD: International classification of diseases; ITT: Intention-to-Treat; MRI: Magnetic resonance tomography; SOP: Standardized operating procedures; TMF: Technologies, Methods and Infrastructure for Networked Medical Research}

\section{Acknowledgements}

We would like to thank Judith Becher, Janina Behrens, Kerstin Bollweg, Fritz Feistner, Robert Fleischmann, Ramanan Ganeshan, Veronika Gonstein, Claudia Gorski, Ulrike Hopf, Anne Keysers, Katharina Kleinsteuber, Malgorzata Kotlarz,
Janine Krätzschmann, Katharina Krimmer, Jana Krismann, Alexander Kunz, Birgit Landes-Fritzsche, Thomas Liman, Christine Omerzu, Christine Ottersbach, Daniel Peters, Ludwig Schlemm, Britta Seidel, Kirsten Stangenberg-Gliss, Anja Smidt, Carl Witt, Christian Wollboldt, for helping to conduct the study.

\section{Consent to publication}

Not applicable.

\section{Standards of reporting}

The protocol of the ANNOTEM study adheres to the reporting guidelines defined in the SPIRIT 2013 Statement [19].

\section{Authors' contributions \\ HJA and ME developed the study concept and idea and secured the funding. HJA is the principle investigator. JEW participated in the design of the study and drafted the manuscript. SE, FIK, ST and TK prepared the statistical analysis plan. AA, HA, HE, FIK and ST contributed relevantly to the preparation of the first manuscript and revised it critically for important intellectual content. AA, KB, AF, HE, CG, SK, KK, IS, and JEW participated in the development and implementation of the network. All authors reviewed the draft and provided feedback, and approved the final manuscript. All authors agree to be accountable for all aspects of the work.}

\section{Funding}

The project is funded by a grant within the framework of the funding line "new types of care" of the Innovation Fund and the Innovation Committee of the Federal Joint Committee (01NVF16028). The funding body is not involved in the design of the study, collection, analysis, and interpretation of data and in writing the manuscript. Open access funding provided by Projekt DEAL

\section{Availability of data and materials Not applicable.}

\section{Ethics approval and consent to participate}

The ANNOTeM study has been approved by the ethics committee (Charité Campus Mitte; Berlin/Germany; no. EA1/078/10 and subsequently by the responsible ethics committees in Brandenburg (AS 90(bB)/2018) and Mecklenburg-Western Pomerania (BB 057/18)) and the Data Protection Department of the Charité. Any changes to the study protocol, must be approved by the Ethics Committee before implementation. According to the positive votes of the ethics committees, informed consent for participation in this study is not necessary.

Within ANNOTeM, the analysis of outcomes is carried out on the basis of health insurance data. A formal approval of the participating hospitals or individual patients for using data of the health insurances is not necessary. The evaluation of the data is based exclusively on anonymised data, which are therefore no longer subject to the General Data Protection Rights (GDPR). The release of the data of the health insurance companies for this purpose was approved by the supervisory authority of the health insurance companies. It is registered at German Clinical Trials Register (DRKS00013067, date of registration: November 16th, 2017, URL: http://www.drks.de/ DRKS00013068). The study will be conducted in accordance with the Declaration of Helsinki in its current version, the guidelines of the International Conference on Harmonization of Good Clinical Practice $(\mathrm{ICH}-$ G(P), and the applicable German laws.

\section{Competing interests}

AA, KB, HE, SE, CG, FIK, SK, KK, IS, ST, and JEW report no disclosures. $\mathrm{ME}$ reports grants from Bayer and fees paid to the Charite from Amgen, Bayer, Boehringer Ingelheim, Bristol-Myers Squibb, Daichi Sankyo, Novartis, Pfizer, Sanofi, all outside of this work.

AF has received consulting fees from Bayer, Roche, Novartis, Biogen Idec, and NovagoTherapeutics, and honoraria for oral presentations from Novartis, Böhringer-Ingelheim, Eli-Lilly and Company, Biogen Idec, Paul-MartiniStiftung, and Daiichi-Sankyo.

TK reports having contributed to an advisory board of CoLucid and a research project funded by Amgen, for which the Charité -

Universitätsmedizin Berlin received an unrestricted compensation. He further reports having received honoraria from Lilly, Newsenselab, and Total for providing methodological advice, from Novartis and from Daiichi Sankyo for 
providing a lecture on neuroepidemiology and research methods, and from the BMJ for editorial services. HJA reports speaker fees and consultancy honoraria received during the conduct of the study from Bayer Vital, Boehringer Ingelheim Pharma, Bristol-Myers-Squibb, Novo Nordisk and Pfizer.

\section{Author details}

${ }^{1}$ Klinik und Hochschulambulanz für Neurologie, Charité - Universitätsmedizin Berlin, Hindenburgdamm 30, D-12203 Berlin, Germany. ${ }^{2}$ Center for Stroke Research Berlin (CSB), Charité - Universitätsmedizin Berlin, Berlin, Germany. ${ }^{3}$ Clinical Research Unit, Berlin Institute of Health, Berlin, Germany. ${ }^{4}$ Department of Neurology, University Medicine Greifswald, Greifswald, Germany. ${ }^{5}$ German Center for Neurodegenerative Diseases, partner site, Rostock, Greifswald, Germany. ${ }^{6}$ Institute of Public Health, Charité Universitätsmedizin Berlin, Berlin, Germany. ${ }^{7}$ Institute for Biometry and Clinical Epidemiology, Charité - Universitätsmedizin Berlin, Berlin, Germany. ${ }^{8}$ Unfallkrankenhaus Berlin, Berlin, Germany. ${ }^{9}$ Excellence Cluster NeuroCure, Berlin, Germany. ${ }^{10}$ German Center for Neurodegenerative Diseases (DZNE), partner site Berlin, Berlin, Germany. ${ }^{11}$ German Centre for Cardiovascular Research (DZHK), partner site Berlin, Berlin, Germany.

Received: 22 June 2020 Accepted: 23 July 2020

Published online: 17 August 2020

\section{References}

1. Emberson J, Lees KR, Lyden P, Blackwell L, Albers G, Bluhmki E, Brott T, Cohen G, Davis S, Donnan G, et al. Effect of treatment delay, age, and stroke severity on the effects of intravenous thrombolysis with alteplase for acute ischaemic stroke: a meta-analysis of individual patient data from randomised trials. Lancet. 2014;384:1929-35.

2. Goyal M, Menon BK, van Zwam WH, Dippel DW, Mitchell PJ, Demchuk AM, Davalos A, Majoie CB, van der Lugt A, de Miquel MA, et al. Endovascular thrombectomy after large-vessel ischaemic stroke: a meta-analysis of individual patient data from five randomised trials. Lancet. 2016;387: 1723-31.

3. Foreman B, Hirsch LJ. Epilepsy emergencies: diagnosis and management. Neurol Clin. 2012;30:11-41 vii.

4. Bodilsen J, Dalager-Pedersen M, Schonheyder HC, Nielsen H. Time to antibiotic therapy and outcome in bacterial meningitis: a Danish population-based cohort study. BMC Infect Dis. 2016;16:392.

5. Fehlings MG, Tetreault LA, Wilson JR, Kwon BK, Burns AS, Martin AR, Hawryluk G, Harrop JS. A clinical practice guideline for the Management of Acute Spinal Cord Injury: introduction, rationale, and scope. Global Spine J. 2017;7:845-94S.

6. DALYs GBD, Collaborators H, Murray CJ, Barber RM, Foreman KJ, Abbasoglu Ozgoren A, Abd-Allah F, Abera SF, Aboyans V, Abraham JP, et al. Global, regional, and national disability-adjusted life years (DALYs) for 306 diseases and injuries and healthy life expectancy (HALE) for 188 countries, 19902013: quantifying the epidemiological transition. Lancet. 2015;386:2145-91.

7. Hatcher-Martin JM, Adams JL, Anderson ER, Bove R, Burrus TM, Chehrenama M, Dolan O'Brien M, Eliashiv DS, Erten-Lyons D, Giesser BS, et al. Telemedicine in neurology: telemedicine work Group of the American Academy of neurology update. Neurology. 2020;94:30-8.

8. Schwamm LH, Holloway RG, Amarenco P, Audebert HJ, Bakas T, Chumbler NR, Handschu R, Jauch EC, Knight WA, Levine SR, et al. A review of the evidence for the use of telemedicine within stroke systems of care: a scientific statement from the American Heart Association/American Stroke Association. Stroke. 2009;40:2616-34.

9. Handschu R, Littmann R, Reulbach U, Gaul C, Heckmann JG, Neundorfer B, Scibor M. Telemedicine in emergency evaluation of acute stroke: interrater agreement in remote video examination with a novel multimedia system. Stroke. 2003;34:2842-6.

10. Audebert HJ, Kukla C, Clarmann von Claranau S, Kuhn J, Vatankhah B, Schenkel J, Ickenstein GW, Haberl RL, Horn M. Telemedicine for safe and extended use of thrombolysis in stroke: the Telemedic pilot project for integrative stroke care (TEMPiS) in Bavaria. Stroke. 2005;36:287-91.

11. Meyer BC, Raman R, Hemmen T, Obler R, Zivin JA, Rao R, Thomas RG, Lyden PD. Efficacy of site-independent telemedicine in the STRokE DOC trial: a randomised, blinded, prospective study. Lancet Neurol. 2008;7:787-95.

12. Schwab S, Vatankhah B, Kukla C, Hauchwitz M, Bogdahn U, Furst A, Audebert HJ, Horn M, Group TE. Long-term outcome after thrombolysis in telemedical stroke care. Neurology. 2007;69:898-903.
13. Schenkel J, Reitmeir P, Von Reden S, Holle R, Boy S, Haberl R, Audebert H. Cost analysis of telemedical treatment of stroke. Gesundheitswesen. 2013; 75:405-12.

14. Nabavi DG, Koennecke HC, Ossenbrink M, Grau A, Busse O, die Stroke Unit $K$, den Zertifizierungsausschuss der DSG, den Vorstand der DSG, den Zertifizierungsausschuss der DSG. Certification criteria for stroke units in Germany : update 2018. Nervenarzt. 2019;90:335-42.

15. Braun M, Schmidt WU, Mockel M, Romer M, Ploner CJ, Lindner T. Coma of unknown origin in the emergency department: implementation of an inhouse management routine. Scand J Trauma Resusc Emerg Med. 2016;24:61.

16. Standardisierte optimierte Prozeduren (SOP) in der Schlaganfallbehandlung [http://www.tempis.de/index.php/sop-druckversion/finish/1-sop/535-tempissop-2015-druckversionpdf.html?25a99fb6f5cd3a21 c61698db179a5bfa=2001 ccda4ae3c6e221909eee46995a94].

17. Sachverständigenrat zur Begutachtung der Entwicklung im Gesundheitswesen (2014) Gutachten 2014 des Sachverständigenrates zur Begutachtung der Entwicklung im Gesundheitswesen. Bedarfsgerechte Versorgung - Perspektiven für ländliche Regionen und ausgewählte Leistungsbereiche 2014.

18. Audebert HJ, Schultes K, Tietz V, Heuschmann PU, Bogdahn U, Haberl RL, Schenkel J. Telemedical project for integrative stroke C: long-term effects of specialized stroke care with telemedicine support in community hospitals on behalf of the Telemedical project for integrative stroke care (TEMPiS). Stroke. 2009;40:902-8.

19. Chan AW, Tetzlaff JM, Altman DG, Laupacis A, Gotzsche PC, Krleza-Jeric K, Hrobjartsson A, Mann H, Dickersin K, Berlin JA, et al. SPIRIT 2013 Statement: defining standard protocol items for clinical trials. Ann Intern Med. 2013;158: 200-7.

\section{Publisher's Note}

Springer Nature remains neutral with regard to jurisdictional claims in published maps and institutional affiliations.

Ready to submit your research? Choose BMC and benefit from:

- fast, convenient online submission

- thorough peer review by experienced researchers in your field

- rapid publication on acceptance

- support for research data, including large and complex data types

- gold Open Access which fosters wider collaboration and increased citations

- maximum visibility for your research: over $100 \mathrm{M}$ website views per year

At BMC, research is always in progress.

Learn more biomedcentral.com/submissions 